\title{
LncRNA TUG1 promotes Ewing's sarcoma cell proliferation, migration, and invasion via the miR-199a-3p-MSI2 signaling pathway
}

\author{
Hu LI, Fang HUANG, Xu-Qiang LIU, Hu-Cheng LIU, Min DAI, Jin ZENG* \\ Department of Orthopedics, The First Affiliated Hospital of Nanchang University, Nanchang, Jiangxi, China \\ *Correspondence: 107891855@qq.com
}

Received November 10, 2020 / Accepted February 3, 2021

\begin{abstract}
The aim of this study was to investigate the roles and potential mechanisms of long non-coding RNA (lncRNA) taurine upregulated gene 1 (TUG1) in the proliferation, migration, and invasion of Ewing's sarcoma cells. RT-qPCR was used to detect the expression of TUG1, microRNA-199a-3p (miR-199a-3p), and musashi2 (MSI2) in Ewing's sarcoma tissues and cell lines. Kaplan-Meier overall survival curves showed the survival rates of Ewing's sarcoma patients with high and low expression of TUG1. The association between the expressions of TUG1/MSI2 and miR-199a-3p in Ewing's sarcoma tissues was assessed by Pearson's correlation analysis. Cell proliferation, migration, and invasion were detected by CCK- 8 assay and Transwell assay, respectively. The protein level of MSI2 was determined using western blotting. The interaction between TUG1/MSI2 and miR-199a-3p was validated by the dual-luciferase reporter assay. The levels of TUG1 and MSI2 were increased, while the level of miR-199a-3p was decreased in Ewing's sarcoma tissues and cells. High expression of TUG1 or MSI2 indicated a decreased overall survival rate of Ewing's sarcoma patients. TUG1/MSI2 level was negatively correlated with miR-199a-3p level. While TUG1 level was positively correlated with MSI2 level. In Ewing's sarcoma cells, knockdown of TUG1/MSI2 or overexpression of miR-199a-3p inhibited cell proliferation, migration, and invasion, whereas the overexpression of TUG1/MSI2 presented the opposite results. TUG1 functioned as a competing endogenous RNA to regulate MSI2 expression by sponging miR-199a-3p. Finally, miR-199a-3p inhibitor or MSI2 overexpression counteracted the TUG1 knockdown-mediated inhibitory effect on Ewing's sarcoma cell proliferation, migration, and invasion. TUG1 promotes proliferation, migration, and invasion of Ewing's sarcoma cells via sequestering miR-199a-3p to enhance the MSI2 expression, suggesting that TUG1 might be a potential target for treating Ewing's sarcoma.
\end{abstract}

Key words: Ewing's sarcoma, TUG1, miR-34a, SIRT1

Ewing's sarcoma is a malignant bone or soft tissue tumor that mostly affects children and young people [1]. It is a small round cell sarcoma with distinct pathological molecular features (different levels of neuroectodermal differentiation). Currently, surgical resection, local radiotherapy, and intensive multidrug chemotherapy are the main treatments $[2,3]$. Due to a pathognomonic chromosomal translocation, the fusion of two genes, EWS and FLI1 occur in Ewing's sarcoma [4]. The fusion protein product EWS-FLI1 plays crucial role in transcription and splicing, which contributes to tumor maintenance [5]. Currently, EWS-FLI1 has been recognized as a potential therapeutic target for Ewing's sarcoma [5]. According to statistics, about $25 \%$ of patients experienced clinically detectable metastases at the time of initial diagnosis, and almost all patients might have the possibility of micro-metastasis during the treatment [6]. Even worse, about $20-25 \%$ of patients with metastasis are usually resistant to intensive multidrug chemotherapy [1].
The long-term survival rate of patients with the metastatic or recurrent disease remains unacceptably low $[1,6,7]$. Even for patients who are cured, the morbidity is substantial after long-term treatment with cytotoxic drugs, so it is extremely necessary to explore new therapeutic strategies for this disease [8].

Musashi2 (MSI2), as a translational regulator, participates in the regulation of hematopoietic stem cell function, self-renewal, and asymmetric cell division, and so on [9-11]. The abnormal high expression of MSI2 has been confirmed in multiple tumors, such as gastric cancer [12], glioblastoma [13], cervical cancer [14], esophageal squamous cell carcinoma [15], etc., which functions as the oncogenic factor driving the initiation and progression of these tumors. Yang et al. showed in vitro and in vivo evidence that MSI2 silencing repressed metastasis of malignant peripheral nerve sheath tumor [16]. Besides, the ablation of MSI2 restrained migration and invasive abilities of bladder cancer cells 
[17]. However, the expression and role of MSI2 in Ewing's sarcoma remains ambiguous.

Long non-coding RNAs (lncRNAs) are a group of transcribed non-coding RNAs that are more than 200 $n t$ in length and do not encode any protein $[18,19]$. It is well established that a $7.1 \mathrm{~kb}$ long lncRNA taurine upregulated gene 1 (TUG1) is a direct transcriptional target of p53, which is activated by p53 response elements in its promoter region [20]. It has been reported that TUG1 was significantly increased in cancer, and TUG1 knockdown suppressed cell proliferation and promoted apoptosis [20]. TUG1 has been considered to be a biomarker and novel therapeutic target for the diagnosis and prognosis of different types of cancers [21-23]. However, the expression and biological functions of TUG1 in Ewing's sarcoma have not been reported. LncRNAs have been verified to crosstalk with functional proteins via sponging microRNAs (miRNAs) [24]. miRNAs are a new class of small regulatory RNAs [25] and also potential biomarkers for cancer diagnosis. There are different miRNA profiles in various tumors, which can be used as molecular biomarkers for tumor prediction, diagnosis, and prognosis [26-28]. A recent study showed that miR-199a-3p functioned as a tumor suppressor to reverse Ewing's sarcoma malignancy [29]. Importantly, miR-199a-3p was predicted to bind to both TUG1 and MSI2. Therefore, we speculated that TUG1 might affect the malignant phenotypes of Ewing's sarcoma cells via regulating the miR-199a-3p/MSI2 axis.

To understand the key roles of the IncRNA-miRNAmRNA functional network in Ewing's sarcoma, in this study we intended to explore whether TUG1 regulated growth, migration, and invasion of Ewing's sarcoma cells through the miR-199a-3p/MSI2 axis. These novel findings might help to uncover the underlying mechanism and provide the potential targets for the treatment of Ewing's sarcoma.

\section{Patients and methods}

Human tissue specimens and cell lines. A total of 52 paired Ewing's sarcoma tissues and adjacent non-tumorous tissues were collected from patients of the First Affiliated Hospital of Nanchang University. The diagnosis was based on EWS-FLI1/ERG classic clinical, radiological, cytopathological, and histopathological findings. The protocol has been approved by the First Affiliated Hospital of Nanchang University. All patients received written consent from the First Affiliated Hospital of Nanchang University ethics committee (No. 2017-03-12). Human normal osteoblast cell line hFOB 1.19 and human Ewing's sarcoma cell line A673 were cultured in Dulbecco's Modified Eagle Medium (DMEM; HyClone; GE Healthcare, Logan, UT, USA) supplemented with $10 \%$ fetal calf serum (FBS; Gibco; Thermo Fisher Scientific). Human Ewing's sarcoma cell lines RD-ES, SK-ES-1, SK-N-MC, and WE-68 were cultured in RPMI-1640 medium (HyClone) supplemented with 15\% FBS. All cell lines were purchased from ATCC (Manassas, VA) and maintained at $37^{\circ} \mathrm{C}$ in a humidified chamber containing $5 \% \mathrm{CO}_{2}$.

Cell transfection. The miR-199a-3p mimics, miR-199a-3p inhibitor, TUG1-short hairpin RNA (shRNA), MSI2-shRNA, and negative control (NC) shRNA were synthesized by Shanghai GenePharma (Shanghai, China). TUG1 and MSI2-overexpression plasmids and empty vector were purchased from Sangon (Shanghai, China). After $24 \mathrm{~h}$ of inoculation, RD-ES and SK-ES-1 cells were transfected with miR-199a-3p mimics, miR-199a-3p inhibitor, TUG1-shRNA, or MSI2-shRNA complexed with Lipofectamine 2000 (Invitrogen) according to the manufacturer's instructions. Cell lysates were harvested after $48 \mathrm{~h}$.

Reverse transcription quantitative real-time polymerase chain reaction (RT-qPCR). To analyze the expression of TUG1, MSI2, and miR-199a-3p in Ewing's sarcoma, total RNA was isolated from cells and surgical specimens according to the protocol of the Total RNA Isolation Kit (Ambion, Austin, TX, USA). After gel electrophoresis verification of RNA integrity, total RNA was reverse-transcribed using the FirstStrand cDNA Synthesis kit (Invitrogen, Carlsbad, CA, USA) with the specific primers. qPCR was then performed on the ABI PRISM 7900 Sequence Detection System (Applied Biosystems, Carlsbad, Calif., USA) using the Quanti-Tect SYBR Green RT-PCR mix to quantify the relative expression of TUG1, MSI2, and miR-199a-3p. GAPDH and U6 snRNA were chosen as a reference for normalizing expression levels, respectively. Primer sequences were as follows: TUG1: forward primer: ACGACTGAGCAAGCACTACC; reverse primer: CTCAGCAATCAGGAGGCACA. miR-199a-3p: forward primer: GGAGGTTTGTTTGTAAGCATGAA; reverse primer: AATGAAGAGAGCACCTATGACAA. MSI2: forward primer: ACGACTCCCAGCACGACC; reverse primer: GCCAGCTCAGTCCACCGTA. GAPDH: forward primer: CTGACTTCAACAGCGACACC; reverse primer: GTGGTCCAGGGGTCTTACTC. U6: forward primer: CTCGCTTCGGCAGCACA; reverse primer: AACGCTTCACGAATTTGCGT.

Cell counting kit-8 (CCK-8) for cell viability. The cells were seeded into 96-well plates in an amount of $1 \times 10^{3} /$ well. After culturing the cells for $0 \mathrm{~h}, 24 \mathrm{~h}, 48 \mathrm{~h}$, and $72 \mathrm{~h}, 10 \mu \mathrm{l}$ of CCK-8 (Dojindo Laboratories, Kumamoto, Japan) reagent was added to each well. The optical density value at a wavelength of $490 \mathrm{~nm}$ was measured using a microplate reader after $2 \mathrm{~h}$.

Transwell assay. Transwell assay was performed using a Transwell chamber (Corning Inc., Corning, NY, USA) with or without Matrigel (BD Biosciences, Bedford, MA, USA). At $24 \mathrm{~h}$ after the transfection, cells in culture medium without FBS $\left(1 \times 10^{4} /\right.$ well $)$ were added to the upper compartment (Millipore, Billerica, MA, USA), while a medium with $10 \%$ FBS was added to the bottom chamber. The cells in the upper compartment were cleared after $24 \mathrm{~h}$ of incubation in the incubator. The migrated or invaded cells were fixed with methanol, stained with $0.5 \%$ crystal violet, and 
digitally photographed and counted under a microscope (IX71, Olympus, Tokyo, Japan). Each sample was assayed in triplicate.

Western blotting. After $48 \mathrm{~h}$ of transfection, the samples were collected in ice-cold PBS, and total protein was extracted from the cells using RIPA buffer. The protein concentration of whole-cell lysates was quantified using a BCA assay kit (Beyotime, Jiangsu, China). Proteins $(20-30 \mu \mathrm{g})$ were separated by SDS-polyacrylamide gel electrophoresis. The separated protein was subsequently transferred to a polyvinylidene fluoride membrane (Millipore). The membrane was then blocked with 5\% skim milk and MSI2 (bsm-52487R; 1:1,000; Bioss, Beijing, China) and GAPDH (TA-O8; 1:2,000, ZSGB-BIO, Beijing, China) antibodies were incubated overnight at $4{ }^{\circ} \mathrm{C}$. After washing in Tris-buffered saline-Tween 20 , membranes were incubated with horseradish peroxidaseconjugated goat anti-rabbit IgG antibody (bs-0295G-HRP, 1:2,000; Bioss). Proteins were visualized by a Chemiluminescence kit (Millipore) according to the manufacturer's recommendations. GAPDH was used as a control.

Dual-luciferase reporter assay. The wild-type TUG1 sequences and MSI2 3' UTR sequences containing the predicted binding sites for miR-199a-3p, or mutated TUG1 sequences and MSI2 3' UTR sequences without miR-199a-3p binding sites were synthesized. The above sequences were inserted into pGL3-Basic Vector (Promega, Madison, WI, USA) and named as pGL3-TUG1-WT, pGL3-MSI2 3' UTR-WT, pGL3-TUG1-MUT, and pGL3-MSI2 3' UTR-MUT, respectively. 293T cells were seeded in 96-well

Table 1. Association of TUG1 expression with clinicopathological features of Ewing's sarcoma.

\begin{tabular}{lcccc}
\hline \multirow{2}{*}{ Features } & \multirow{2}{*}{$\begin{array}{c}\text { Number of } \\
\text { cases }\end{array}$} & \multicolumn{2}{c}{ TUG1 } & \multirow{2}{*}{ p-value } \\
\cline { 3 - 4 } & & & & \\
Age at diagnosis & 26 & 12 & 14 & 0.614 \\
$\quad<18$ & 26 & 11 & 15 & \\
$\quad \geq 18$ & & & & \\
Gender & 22 & 15 & 7 & 0.783 \\
$\quad$ Female & 30 & 18 & 12 & \\
$\quad$ Male & & & & \\
Clinical stage & 24 & 13 & 11 & 0.039 \\
$\quad$ I+IIA & 28 & 22 & 6 & \\
$\quad$ IIB/III & & & & \\
Distant metastasis & 21 & 10 & 11 & 0.012 \\
$\quad$ Absent & 31 & 22 & 9 & \\
$\quad$ Present & & & & \\
Tumor size (cm) & 29 & 16 & 13 & 0.591 \\
$\quad<5$ & 23 & 16 & 7 & \\
$\quad \geq 5$ & & & & \\
Anatomic location & 19 & 11 & 8 & 0.624 \\
$\quad$ Tibia/femur & 33 & 17 & 16 & \\
$\quad$ Elsewhere & &
\end{tabular}

Note: ${ }^{*} \mathrm{p}$-value obtained from Pearson $\chi^{2}$-test or Fisher's exact test plates and then co-transfected with the constructed recombinant luciferase vectors and miR-199a-3p mimic or mimic NC using Lipofectamine 2000 (Invitrogen). Luciferase activity was measured with the Dual-Luciferase Reporter Assay System (Promega) $48 \mathrm{~h}$ after transfection.

Statistical analysis. Data are expressed as mean \pm standard deviation of at least three independent experiments. Statistical analysis was made using Student's t-test, one-way ANOVA with Bonferroni post hoc analysis, Kaplan-Meier with log-rank methods, and Pearson's correlation using SPSS 22.0 software (SPSS Inc., Chicago, IL, USA). A p-value $<0.05$ was considered to be significantly significant.

\section{Results}

TUG1 and MSI2 were upregulated but miR-199a-3p was downregulated in Ewing's sarcoma tissues. We determined the expression level of TUG1 in Ewing's sarcoma tissues compared with corresponding adjacent non-tumorous tissues. The expression level of TUG1 was significantly increased in tumor tissues obtained from 52 patients diagnosed with Ewing's sarcoma compared with the adjacent non-tumorous tissues $(\mathrm{p}<0.01$, Figure $1 \mathrm{~A})$. Kaplan-Meier analysis and log-rank test showed that Ewing's sarcoma patients with a high expression of TUG1 had a decreased overall survival rate $(p<0.05$, Figure $1 B)$. Whereas, the expression level of miR-199a-3p was significantly decreased in Ewing's sarcoma tissues compared with the adjacent non-tumorous tissues $(\mathrm{p}<0.01$, Figure 1C). The results of Pearson correlation analysis revealed that the miR-199a-3p level was found to be negatively correlated with the TUG1 level (Figure 1D). Further correlation analysis confirmed that expression of IncRNA TUG1 was closely correlated with the clinical stage and distant metastasis $(\mathrm{p}<0.05)$, but not with gender, age, tumor size, and anatomic location ( $p>0.05$, Table 1). Moreover, we found an increased expression of MSI2 in Ewing's sarcoma tissues $(\mathrm{p}<0.01)$, which was negatively correlated with miR-199a-3p expression (Figures 1E, 1F). As shown in Figure 1G, high expression of MSI2 indicated a lower overall survival rate of Ewing's sarcoma patients $(\mathrm{p}<0.01)$. There was a positive correlation between TUG1 and MSI2 expression (Figure 1H).

TUG1 affected proliferation, migration, and invasion of Ewing's sarcoma cells. Then, we determined the functional roles of TUG1 in the malignant phenotypes of Ewing's sarcoma cells. As assessed by RT-qPCR, the expression level of TUG1 in Ewing's sarcoma cell lines RD-ES, WE-68, A673, SK-N-MC, and SK-ES-1 was significantly higher than that in normal osteoblast cell line hFOB 1.19 (Figure 2A). To further investigate the effect of TUG1 on Ewing's sarcoma cell proliferation, migration, and invasion, three sh-RNAs targeting TUG1 (1\#TUG1 shRNA, 2\#TUG1 shRNA, and 3\#TUG1 shRNA) and one negative control (non-specific shRNA) were designed, synthesized, and transfected into RD-ES and SK-ES-1 cells with higher TUG1 level. RT-qPCR 

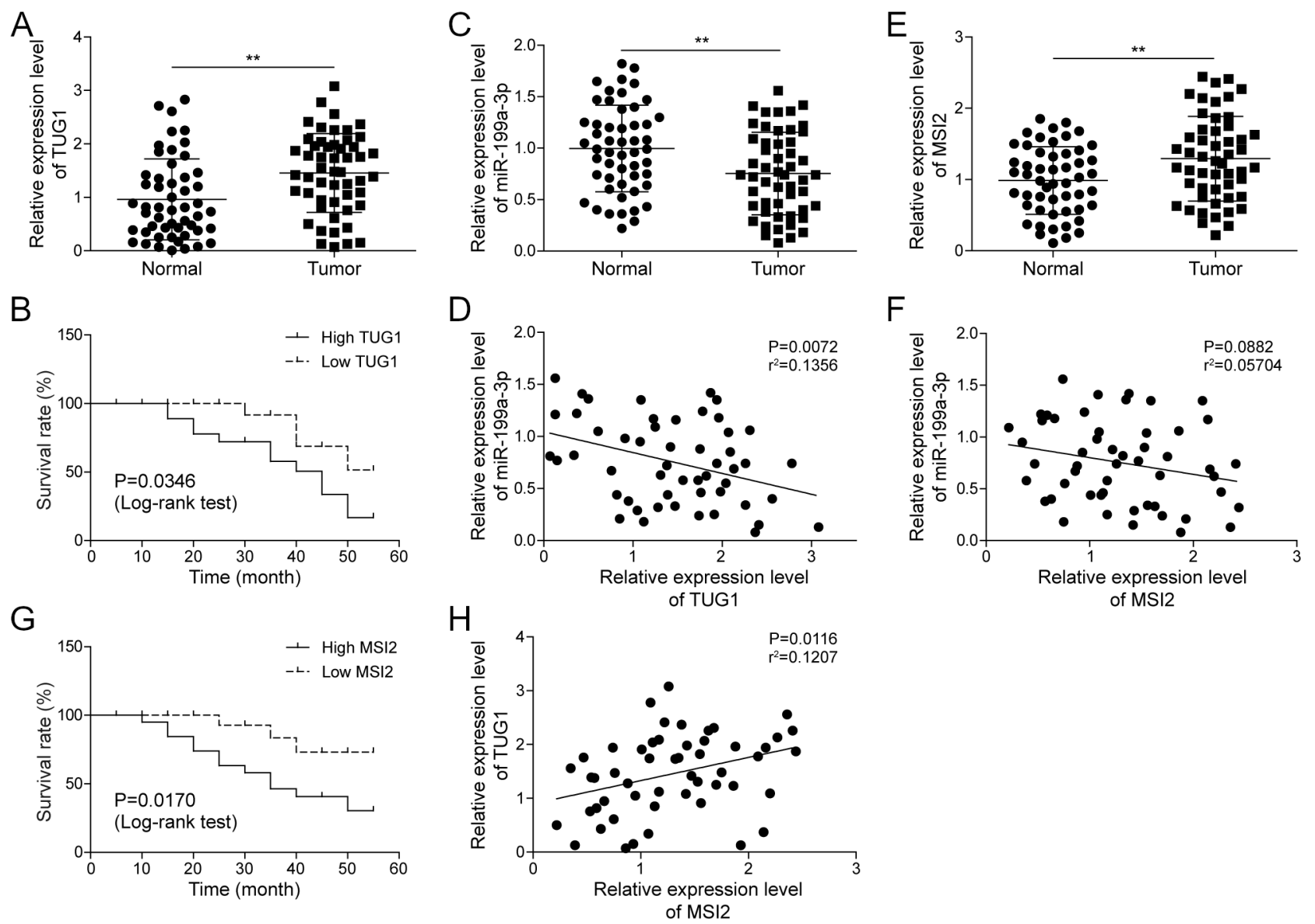

Figure 1. Upregulation of TUG1 and MSI2, while downregulation of miR-199a-3p in Ewing's sarcoma tissues. A) RT-qPCR was used to detect the expression of TUG1 in Ewing's sarcoma tissues and paired non-tumorous tissues $(\mathbf{n}=52)$. B) Kaplan-Meier overall survival curves of Ewing's sarcoma patients with high and low expression of TUG1. C) RT-qPCR for detecting the expression of miR-199a-3p in Ewing's sarcoma tissues and paired noncancerous tissue. D) The association between the expressions of TUG1 and miR-199a-3p was assessed by Pearson's correlation analysis. E) RT-qPCR for determining the expression of MSI2 in Ewing's sarcoma tissues and paired non-cancerous tissue. F) Pearson's correlation analysis for the correlation between miR-199a-3p and MSI2 expression. G) Kaplan-Meier overall survival curves of Ewing's sarcoma patients with high and low expression of MSI2. H) Pearson's correlation analysis for the correlation between TUG1 and MSI2 expression. Data are shown as mean \pm SD from three independent experiments. ${ }^{* *} \mathrm{p}<0.01$

result showed that all these three shRNAs effectively inhibited TUG1 expression in RD-ES and SK-ES-1 cells, among which \#1TUG1 shRNA showed the strongest inhibitory effect and therefore was selected for subsequent experiments (Figure 2B). In addition, RD-ES and SK-ES-1 cells transfected with sh-TUG1 displayed significantly lower proliferation capacity compared with those transfected with sh-NC (Figure 2C). As shown in Figures 2D and 2E, knockdown of TUG1 resulted in the weakened migration and invasive abilities in RD-ES and SK-ES-1 cells. Furthermore, the protein level of MSI2 was also significantly reduced after TUG1 knockdown (Figure 2F). On the contrary, overexpression of TUG1 promoted the proliferation, migration, invasion, and upregulated MSI2 protein level in RD-ES cells (Figures 2G-2J). These findings revealed that TUG1 affected the proliferation, migration, and invasion of Ewing's sarcoma cells.
miR-199a-3p overexpression suppressed proliferation, migration, and invasion of Ewing's sarcoma cells. First, the expression of miR-199a-3p in various Ewing's sarcoma cells was examined. As shown in Figure 3A, the expression of miR-199a-3p in Ewing's sarcoma cell lines was significantly lower than that in hFOB1.19 cells. To further evaluate the biological roles of miR-199a-3p in Ewing's sarcoma cells, miR-199a-3p was overexpressed by transfection with miR-199a-3p mimics in RD-ES and SK-ES- 1 cells $(p<0.01$, Figure $3 \mathrm{~B}$ ). Subsequently, the results of the CCK- 8 assay indicated that the overexpression of miR-199a-3p remarkably inhibited cell proliferation (Figure 3C). Besides, the results of the Transwell assay indicated that miR-199a-3p mimics obviously repressed the migration and invasion of RD-ES and SK-ES-1 cells (Figures 3D, 3E). Western blotting analysis further showed that overexpression of miR-199a-3p downregulated MSI2 protein expression level (Figure 3F). 

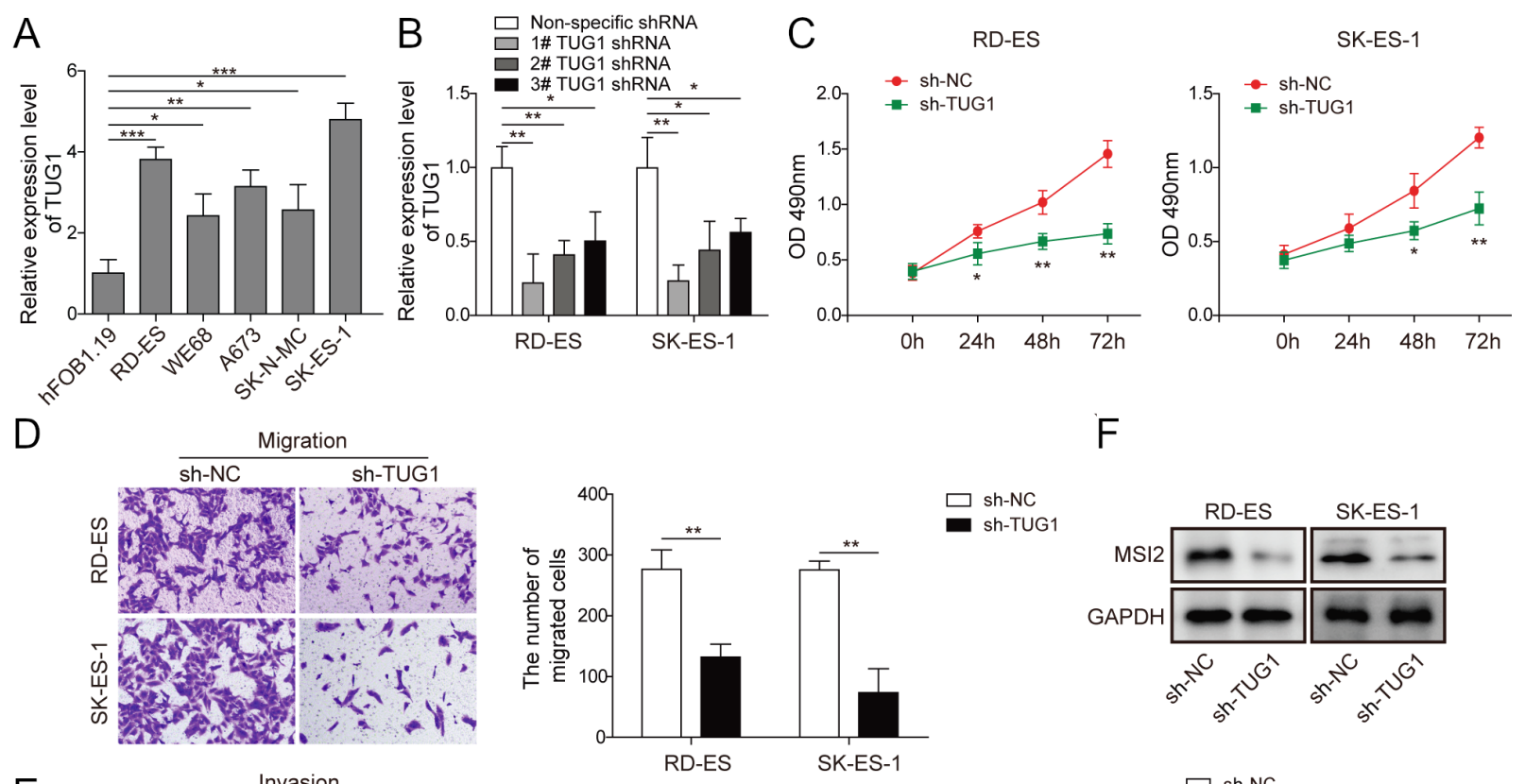

$\mathrm{F}$

$E$
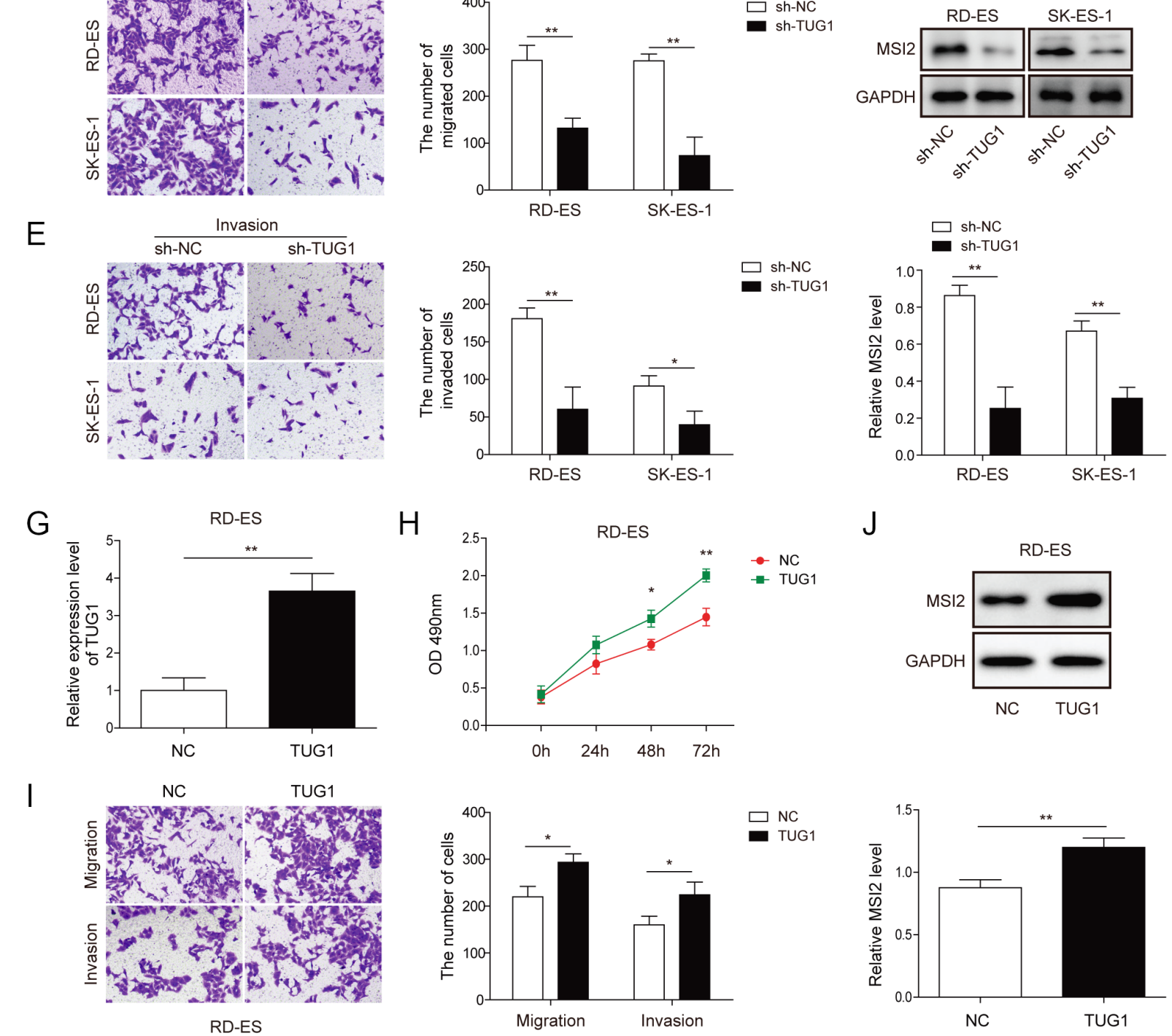

Figure 2. TUG1 knockdown affected proliferation, migration, and invasion of Ewing's sarcoma cells. A) Expression of TUG1 in Ewing's sarcoma cell lines and human normal osteoblast line hFOB1.19 was detected by RT-qPCR. B) RT-qPCR was used to detect TUG1 expression in RD-ES and SKES-1 cells transfected with sh-TUG1 or sh-NC. C) CCK-8 was performed to determine the proliferation of RD-ES and SK-ES-1 cells transfected with sh-TUG1 or sh-NC. D, E) Transwell assay was used to measure migration and invasion abilities of RD-ES and SK-ES-1 cells transfected with sh-TUG1 or sh-NC. F) The protein level of MSI2 in RD-ES and SK-ES-1 cells after transfection with sh-TUG1 or sh-NC was determined by western blotting. G) RT-qPCR for measuring TUG1 level in RD-ES cells. H) CCK-8 for determining the proliferation of RD-ES cells. I) The migration and invasion abilities of RD-ES cells were detected by Transwell assay. J) Western blotting for MSI2 protein level in RD-ES cells. Data are expressed as mean \pm SD from three independent experiments. ${ }^{*} \mathrm{p}<0.05,{ }^{* *} \mathrm{p}<0.01,{ }^{* *} \mathrm{p}<0.001$ 
These data indicated that miR-199a overexpression inhibited proliferation, migration, and invasion of Ewing's sarcoma cells.

MSI2 regulated the proliferation, migration, and invasion of Ewing's sarcoma cells. Accordingly, upregulation of MSI2 level in multiple Ewing's sarcoma cells compared with hFOB1.19 cells was confirmed by western blotting
(Figure 4A). In addition, MSI2-shRNA-mediated silencing of MSI2 in RD-ES and SK-ES-1 cells was verified by western blotting $(\mathrm{p}<0.01$, Figure $4 \mathrm{~B})$. Moreover, the proliferation, migration, and invasion of RD-ES and SK-ES-1 cells were evidently repressed after MSI2 was knocked down $(\mathrm{p}<0.05$ or $\mathrm{p}<0.01$, Figures $4 \mathrm{C}-4 \mathrm{E})$. However, MSI2 overexpression led to enhanced proliferation, migration, and invasion in RD-ES
A

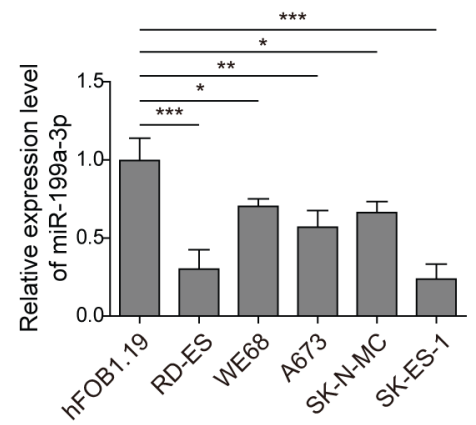

B

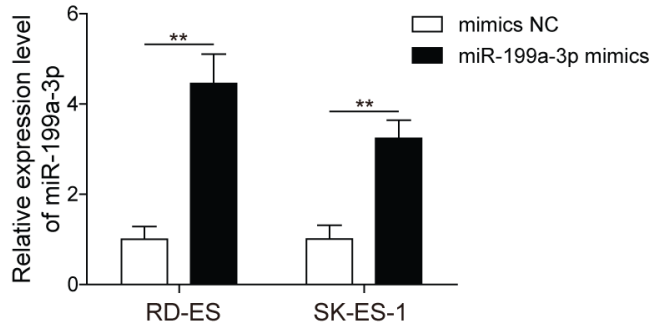

C
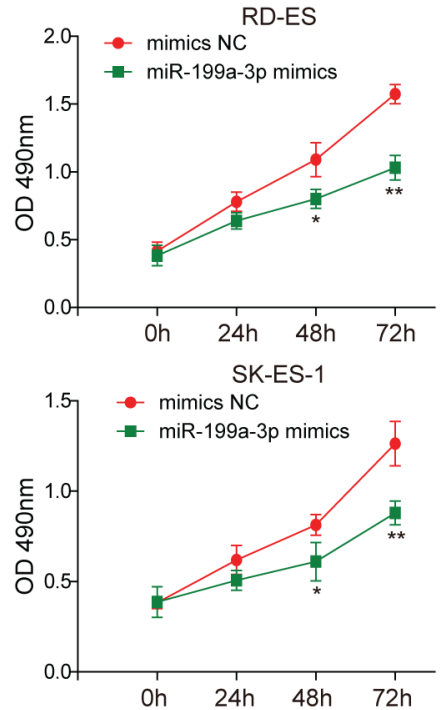

$\mathrm{F}$

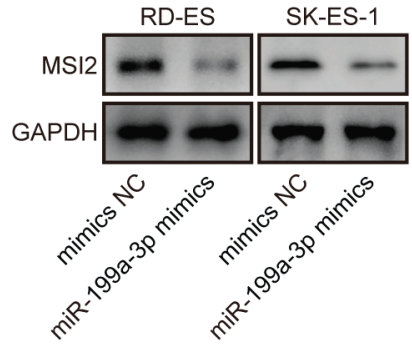

D
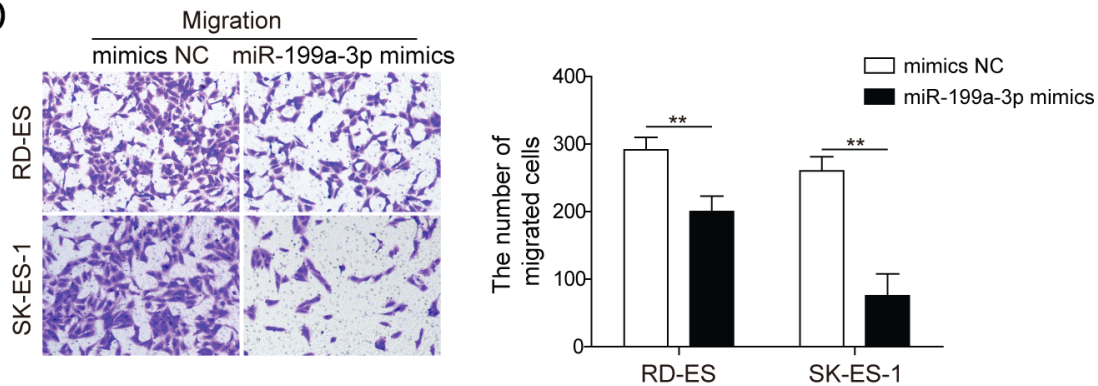

E
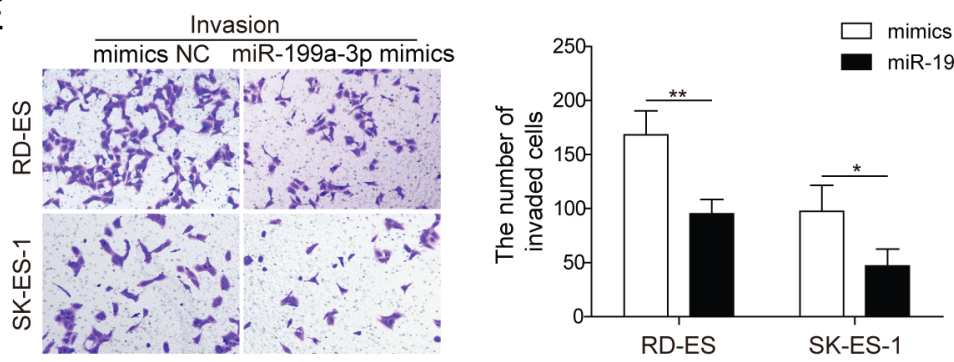

Figure 3. miR-199a-3p overexpression suppressed proliferation, migration, and invasion of Ewing's sarcoma cells. A) Expression of miR-199a-3p in Ewing's tumor cell lines and human normal osteoblast cell line hFOB1.19 was detected by RT-qPCR. B) Expression of miR-199a-3p in RD-ES and SKES-1 cells transfected with miR-199a-3p mimics or mimics-NC was detected by RT-qPCR. C) CCK-8 was performed to evaluate the proliferation of RD-ES and SK-ES-1 cells transfected with miR-199a-3p mimics or mimics-NC. D, E) Transwell assay was used to determine the migration and invasion of RD-ES and SK-ES-1 cells. F) Western blotting for measuring the protein levels of MSI2 in RD-ES and SK-ES-1 cells. Data are expressed as mean \pm SD from three independent experiments, ${ }^{*} \mathrm{p}<0.05,{ }^{\star *} \mathrm{p}<0.01,{ }^{\star * *} \mathrm{p}<0.001$ 
A
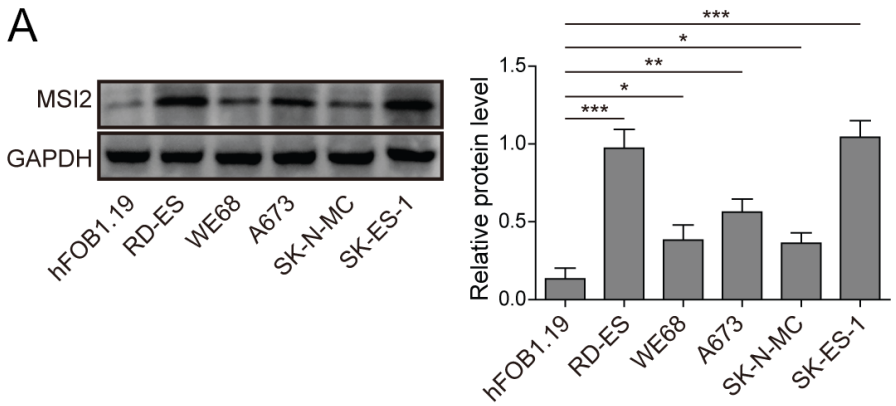
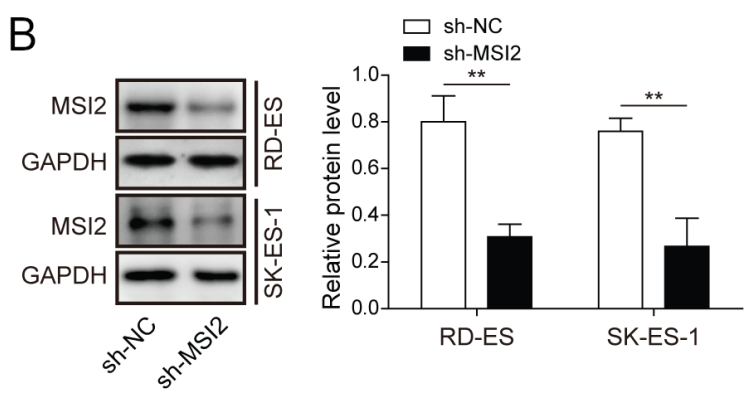

C
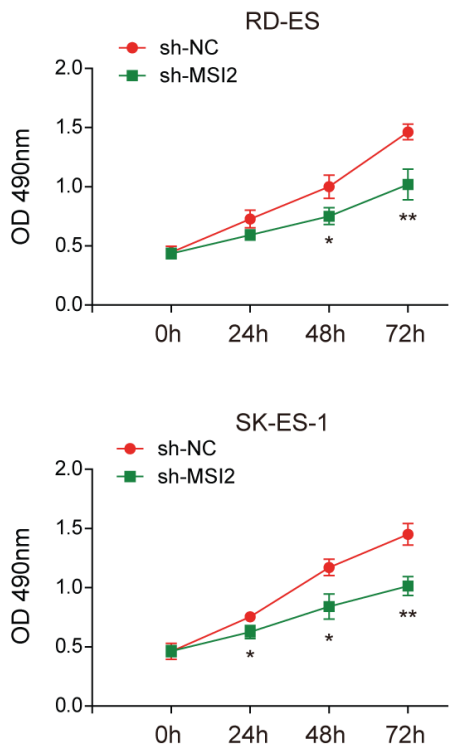

F

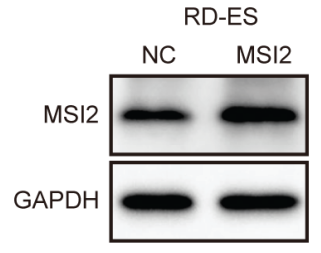

$\mathrm{H}$ RD-ES

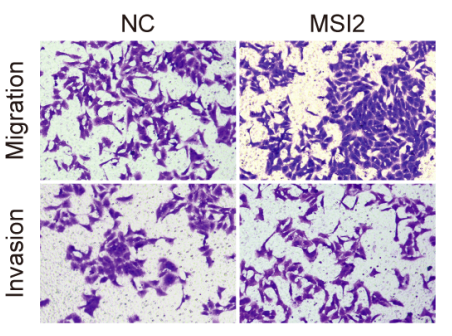

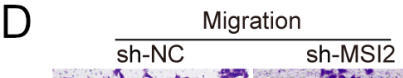
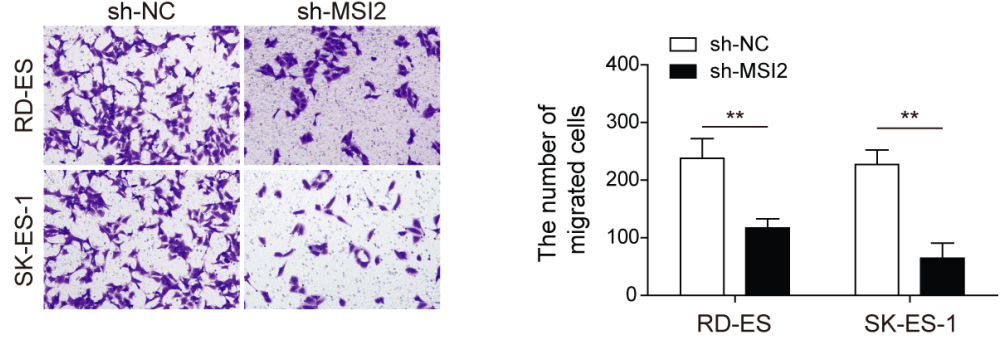

E
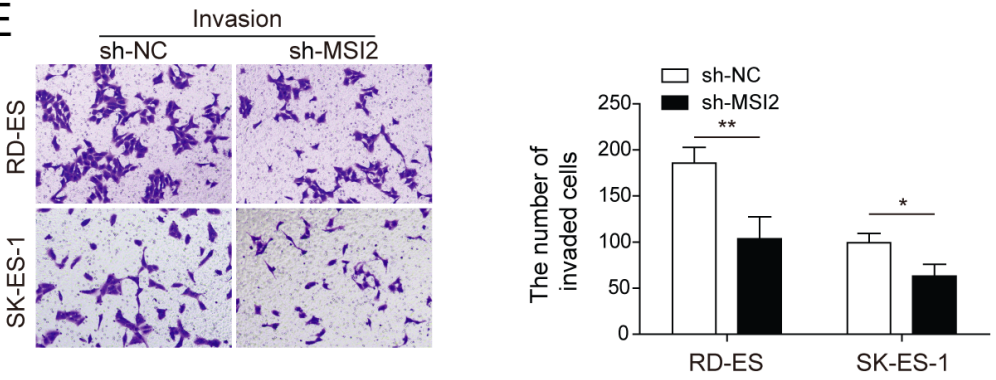

G
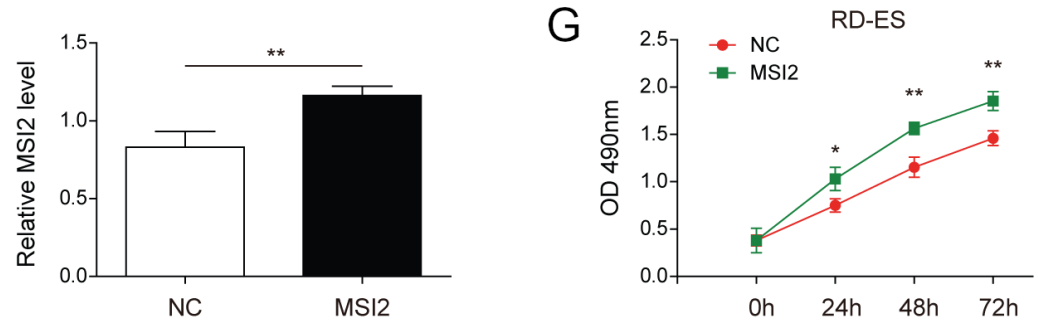

Figure 4. MSI2 regulated proliferation, migration, and invasion of Ewing's sarcoma cells. A) MSI2 protein expression in various Ewing's tumor cell lines and human normal osteoblast cell line hFOB1.19 was assessed by western blotting. B) The protein level of MSI2 in RD-ES and SK-ES-1 cells after transfection with sh-MSI2 or sh-NC was determined by western blotting. C) CCK-8 for detecting the proliferation of RD-ES and SK-ES-1 cells. D, E) The migration and invasive abilities of RD-ES and SK-ES-1 cells were assessed by Transwell assay. F) Western blotting for evaluating the protein level of MSI2 in RD-ES cells. G) The proliferation of RD-ES cells was assessed by CCK-8. H) Transwell assay for determining the migration and invasive abilities of RD-ES cells. Data are expressed as mean \pm SD from three independent experiments, ${ }^{\star} \mathrm{p}<0.05,{ }^{\star *} \mathrm{p}<0.01,{ }^{\star * *} \mathrm{p}<0.001$ 
cells ( $\mathrm{p}<0.05$ or $\mathrm{p}<0.01$, Figures $4 \mathrm{~F}-4 \mathrm{H}$ ). These observations suggested that MSI2 regulated the malignant growth, migration, and invasion of Ewing's sarcoma cells.

TUG1 promoted MSI2 expression by sponging miR-199a-3p. Next, we investigated the potential regulatory mechanisms of TUG1 underlying the malignancy of Ewing's sarcoma cells. As shown in Figure 5A, overexpression of miR-199a-3p strikingly reduced MSI2 mRNA expression $(p<0.05)$, and depletion of TUG1 resulted in a significant increase in miR-199a-3p expression $(p<0.01)$, while a decrease in MSI2 mRNA expression ( $p<0.05$, Figure $5 B)$. Additionally, bioinformatics analysis revealed that TUG1 and the 3'UTR region of MSI2 contained binding sites for miR-199a-3p (Figure 5C). Then, we used a dual-luciferase reporter assay to confirm the direct interaction between TUG1/MSI2 and miR-199a-3p. The results demonstrated that co-transfection with pGL3-TUG1-WT or pGL3-MSI2 3'UTR-WT and miR-199a-3p mimics resulted in an obvious suppression of luciferase activity $(\mathrm{p}<0.01)$, while this suppression was not found in the pGL3-TUG1-MUT or pGL3-MSI2 3'UTR-MUT ( $p>0.05$, Figure 5D) group. Collectively, these data demonstrated the TUG1 promoted MSI2 expression by sponging miR-199a-3p.

TUG1 contributed to Ewing's sarcoma cell proliferation, migration, and invasion via targeting the miR-199a-3p/ MSI2 axis. Finally, we sought to evaluate whether TUG1 affected the biological behaviors of Ewing's sarcoma cells via regulating the miR-199a-3p/MSI2 axis. The results of the CCK-8 assay showed that TUG1 knockdown significantly inhibited the proliferation of RD-ES and SK-ES-1 cells, however, miR-199a-3p inhibitor could reverse this inhibitory effect $(\mathrm{p}<0.01$ or $\mathrm{p}<0.001$, Figure 6A). Similarly, Transwell assay showed that downregulation of TUG1 significantly reduced the migration and invasive capacities of $\mathrm{RD}-\mathrm{ES}$ and SK-ES-1 cells, which were remarkably counteracted by a miR-199a-3p inhibitor $(\mathrm{p}<0.05$ or $\mathrm{p}<0.01$, Figures $6 \mathrm{~B}$, 6C). Furthermore, western blotting analysis suggested that TUG1 knockdown-mediated decrease in MSI2 protein level was partly reversed by the transfection with miR-100a-3p inhibitor $(\mathrm{p}<0.05$ or $\mathrm{p}<0.01$, Figure $6 \mathrm{D})$. Accordingly, TUG1 knockdown-mediated inhibition of proliferation, migration, and invasion was significantly abolished by MSI2 overexpression $(\mathrm{p}<0.01$, Figures $6 \mathrm{E}, 6 \mathrm{~F})$. These data indicated that TUG1 promoted Ewing's sarcoma cell proliferation, migration, and invasion via miR-199a-3p-mediated regulation of the MSI2 signaling pathway.

\section{Discussion}

Ewing's sarcoma, commonly located in the middle portion or in soft tissue of long bones, is the second most common bone malignancy mainly occurring in children
A

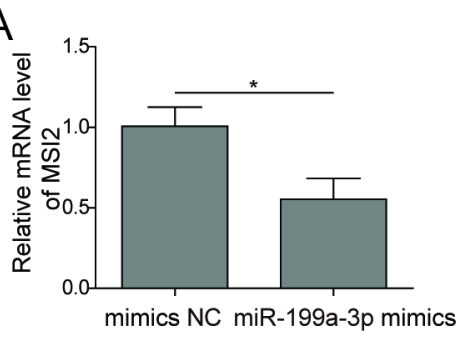

C
$\mathrm{B}$

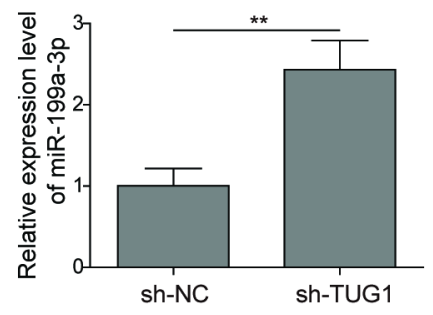

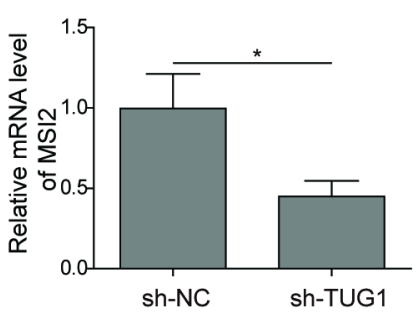
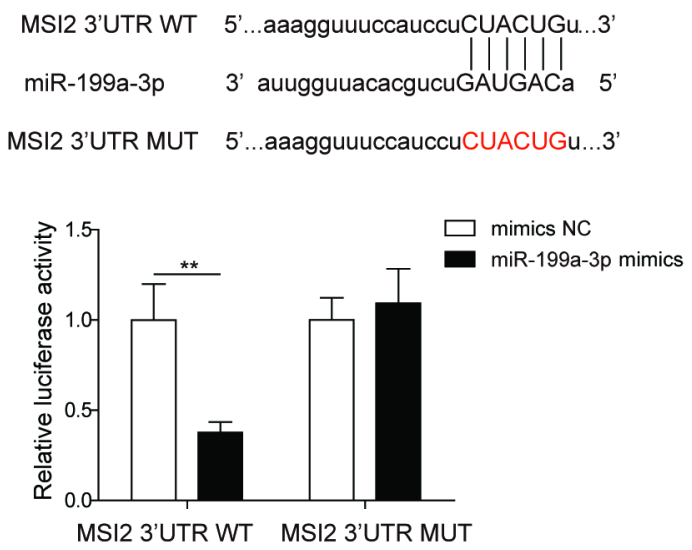

Figure 5. TUG1 promoted MSI2 expression by sponging miR-199a-3p. A) RT-qPCR for detecting the expression of MSI2 after transfection with miR199a-3p mimics or mimics-NC. B) RT-qPCR was used to detect the expression of miR-199a-3p and MSI2 after transfection with sh-TUG1. C) The predicted binding sites between miR-199a-3p and TUG1/MSI2 3'UTR. D) Dual-luciferase reporter assay was carried out to determine the interaction between miR-199a-3p and TUG1/MSI2. Data are expressed as the mean \pm SD from three independent experiments, ${ }^{*} \mathrm{p}<0.05$, ${ }^{\star *} \mathrm{p}<0.01$ 

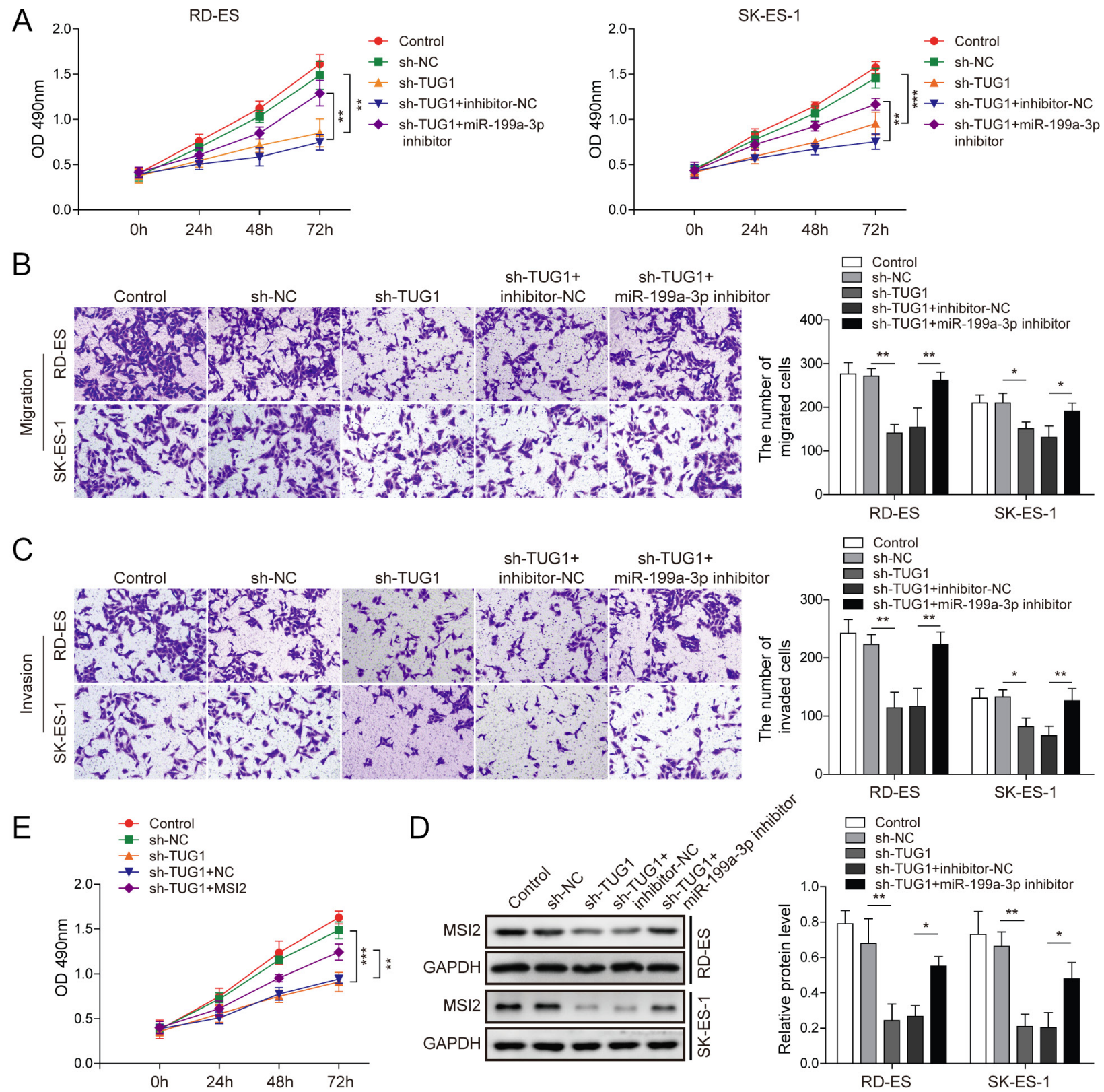

$\mathrm{F}$
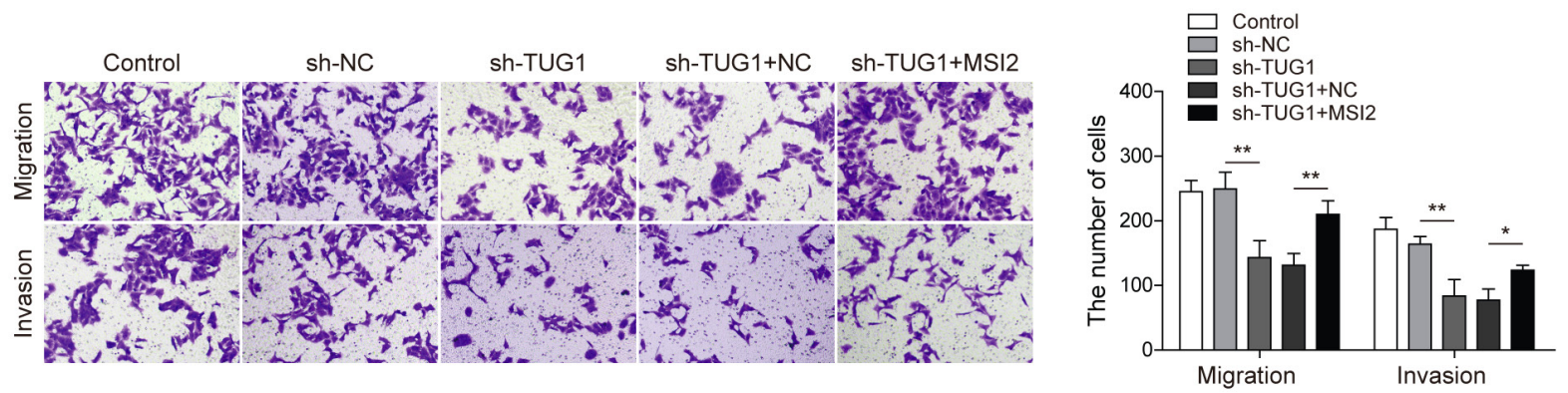

Figure 6. TUG1 contributed to Ewing's sarcoma cell proliferation, migration, and invasion via miR-199a-3p mediated regulation of MSI2. A) CCK-8 was used to determine the proliferation of RD-ES and SK-ES-1 cells from different groups. B, C) Transwell assay was used to determine the migration and invasion of RD-ES and SK-ES-1 cells with various treatments. D) The protein level of MSI2 from various treatment groups was evaluated by western blotting. E) CCK-8 for assessing the proliferation of RD-ES cells from different groups. F) Transwell assay for determining the migration and invasion of RD-ES cells with various treatments. Data are expressed as the mean \pm SD from three independent experiment, ${ }^{*} \mathbf{p}<0.05 ;{ }^{* *} \mathrm{p}<0.01,{ }^{* * *} \mathrm{p}<0.001$ 
and adolescents [30]. In the past decades, the survival rate of Ewing's sarcoma patients has improved, which depends to a large extent on the development of multimodal therapy and supportive care [31]. However, the long-term prognosis of patients with metastatic or recurrent Ewing's sarcoma is still not optimistic [1]. Therefore, uncovering the pathogenesis to identify novel therapeutic measures for Ewing's sarcoma is urgently needed. In the present study, we firstly explored the tumorigenic role of lncRNA TUG1 in Ewing's sarcoma. Our data showed that the interference of TUG1 restrained proliferation, migration, and invasion of Ewing's sarcoma cells. The potential molecular mechanism of TUG1 may be the promotion of the MSI2 expression via sponging miR-199a-3p.

Numerous studies have shown that the dysregulation of lncRNAs may be related to the pathological processes of some human diseases, such as cancer, cardiovascular disease, and central nervous system disease [19, 32]. However, studies on the roles and mechanisms of lncRNAs in Ewing's sarcoma are very limited. LncRNA TUG1 was originally detected in a genomic screen for genes in response to taurine treatment of developing mouse retinal cells [33]. TUG1 has been found to play a crucial regulatory role in various cancer-related biological processes and may provide a new therapeutic regimen in cancer treatment. The results of two previous meta-analyses have shown that increased TUG1 expression is a negative factor in improving human cancer survival, and it is closely related to increased tumor size, pathological stage progression, and distant metastasis [34, 35]. In this study, our research indicated that TUG1 was overexpressed in Ewing's sarcoma tissues and cell lines. On the other hand, inhibition of TUG1 expression reduced Ewing's sarcoma cell proliferation, migration, and invasion, suggesting the pivotal roles of TUG1 in the progression of Ewing's sarcoma.

miR-199a-3p has been widely recognized as a tumor suppressor in multiple types of human cancers. A recent study suggested that miR-199a-3p overexpression halted the carcinogenesis process of esophageal squamous cell carcinoma [36]. In non-small cell lung cancer, miR-199a-3p was found to inhibit stemness and induce mitochondria dysfunction-mediated apoptosis of A549 cells via targeting ZEB1 [37]. Similarly, this study found that the expression level of miR-199a-3p in Ewing's sarcoma tissues and cell lines was significantly lower than that in adjacent non-tumorous tissues and human normal osteoblast cell line, and overexpression of miR-199a-3p could inhibit Ewing's sarcoma cell proliferation, migration, and invasion. MSI2 belongs to the Musashi gene family and is a well-characterized tumor-driver in the tumorigenesis of some human cancers. For instance, in cervical cancer, the aberrant upregulation of MSI2 promoted the aggressive phenotypes of cervical cancer cells [38]. Li et al. found that MSI2 promoted the proliferation and epithelial-mesenchymal transition of esophageal squamous cell carcinoma cells [15]. In the present study, the upregulation of MSI2 was demonstrated in Ewing's sarcoma tissues and cells and contributed to the proliferation, migration, and invasion of cancer cells. More importantly, our data showed that the protein level of MSI2 was reduced by miR-199a-3p mimics in Ewing's sarcoma cells. Further dual-luciferase reporter assay revealed that MSI2 was a target gene of miR-199a-3p. Therefore, the miR-199a-3p/MSI2 axis participated in the pathogenesis of Ewing's sarcoma.

Mounting evidence has demonstrated that lncRNAs weaken the inhibitory effect of miRNA on its target mRNA by acting as a miRNA sponge, and thus play a role in regulating tumor cell proliferation, apoptosis, migration, and invasion [39]. TUG1 has been documented to regulate the development of cancer via affecting target gene expression through sponging miRNA [23]. Wang et al. reported that TUG1 facilitated the proliferation, migration, and invasion of hepatocellular carcinoma cells through upregulation of COL1A1 via sequestering miR-29c-3p [40]. A study by Zhang et al. showed that depletion of TUG1 abated cisplatin resistance of tongue squamous cell carcinoma cells via targeting the miR-133b/CXCR4 axis [41]. In this study, a negative correlation between TUG1/MSI2 and miR-199a-3p in Ewing's sarcoma patient samples was observed. In addition, knockdown of TUG1 led to the increased miR-199a-3p expression, while decreased MSI2 expression in Ewing's sarcoma cells. As expected, the bioinformatic analysis showed that miR-199a-3p could bind to TUG1 and MSI2 3'UTR. Subsequently, the direct interaction between miR-199a-3p and TUG1/MSI2 was demonstrated by luciferase reporter assay. Moreover, the regulatory functions of TUG1 silencing in Ewing's sarcoma cells were abolished by miR-199a-3p inhibitor. Therefore, TUG1 inhibition restrained the proliferation, migration, and invasion of Ewing's sarcoma cells through targeting the miR-199a-3p/MSI2 axis.

In summary, our study demonstrates that TUG1 contributes to the proliferation, migration, and invasion of Ewing's sarcoma cells. Mechanistically, TUG1 sponges miR-199a-3p to increase the MSI2 expression. These findings provide clues to further explore the mechanisms of lncRNAs in the development and progression of Ewing's sarcoma. This novel TUG1-miR-199a-3p-MSI2 network helps to understand the underlying pathogenesis of Ewing's sarcoma, which may identify useful therapeutic targets for Ewing's sarcoma in the future.

Acknowledgments: This work was supported by the National Natural Science Foundation of China (NO. 81860404).

\section{References}

[1] GASPAR N, HAWKINS DS, DIRKSEN U, LEWIS IJ, FERRARI S et al. Ewing Sarcoma: Current Management and Future Approaches Through Collaboration. J Clin Oncol 2015; 33: 3036-3046. https://doi.org/10.1200/JCO.2014.59.5256 
[2] SHEFFIELD NC, PIERRON G, KLUGHAMMER J, DATLINGER P, SCHONEGGER A et al. DNA methylation heterogeneity defines a disease spectrum in Ewing sarcoma. Nat Med 2017; 23: 386-395. https://doi.org/10.1038/ nm. 4273

[3] PAPPO AS, DIRKSEN U. Rhabdomyosarcoma, Ewing Sarcoma, and Other Round Cell Sarcomas. J Clin Oncol 2018; 36: 168-179. https://doi.org/10.1200/JCO.2017.74.7402

[4] KOVAR H. Downstream EWS/FLI1 - upstream Ewing's sarcoma. Genome Med 2010; 2: 8. https://doi.org/10.1186/ gm129

[5] ERKIZAN HV, UVERSKY VN, TORETSKY JA. Oncogenic partnerships: EWS-FLI1 protein interactions initiate key pathways of Ewing's sarcoma. Clin Cancer Res 2010; 16: 4077-4083. https://doi.org/10.1158/1078-0432.CCR-092261

[6] STAHL M, RANFT A, PAULUSSEN M, BOLLING T, VIETH V et al. Risk of recurrence and survival after relapse in patients with Ewing sarcoma. Pediatr Blood Cancer 2011; 57: 549-553. https://doi.org/10.1002/pbc.23040

[7] DE ALAVA E. Ewing Sarcoma, an Update on Molecular Pathology with Therapeutic Implications. Surg Pathol Clin 2017; 10: 575-585. https://doi.org/10.1016/j.path.2017.04.001

[8] GINSBERG JP, GOODMAN P, LEISENRING W, NESS KK, MEYERS PA et al. Long-term survivors of childhood Ewing sarcoma: report from the childhood cancer survivor study. J Natl Cancer Inst 2010; 102: 1272-1283. https://doi. org/10.1093/jnci/djq278

[9] WUEBBEN EL, MALLANNA SK, COX JL, RIZZINO A. Musashi2 is required for the self-renewal and pluripotency of embryonic stem cells. PLoS One 2012; 7: e34827. https:// doi.org/10.1371/journal.pone.0034827

[10] PARK SM, DEERING RP, LU Y, TIVNAN P, LIANOGLOU $S$ et al. Musashi-2 controls cell fate, lineage bias, and TGFbeta signaling in HSCs. J Exp Med 2014; 211: 71-87. https:// doi.org/10.1084/jem.20130736

[11] BYERS RJ, CURRIE T, THOLOULI E, RODIG SJ, KUTOK JL. MSI2 protein expression predicts unfavorable outcome in acute myeloid leukemia. Blood 2011; 118: 2857-2867. https://doi.org/10.1182/blood-2011-04-346767

[12] YANG Z, LI J, SHI Y, LI L, GUO X. Increased musashi 2 expression indicates a poor prognosis and promotes malignant phenotypes in gastric cancer. Oncol Lett 2019; 17: 2599-2606. https://doi.org/10.3892/ol.2019.9889

[13] JIANG X, TAN J, WEN Y, LIU W, WU S et al. MSI2-TGFbeta/TGF-beta R1/SMAD3 positive feedback regulation in glioblastoma. Cancer Chemother Pharmacol 2019; 84: 415425. https://doi.org/10.1007/s00280-019-03892-5

[14] LIU Y, FAN Y, WANG X, HUANG Z, SHI K et al. Musashi-2 is a prognostic marker for the survival of patients with cervical cancer. Oncol Lett 2018; 15: 5425-5432. https://doi. org/10.3892/ol.2018.8077

[15] LI Z, JIN H, MAO G, WU L, GUO Q. Msi2 plays a carcinogenic role in esophageal squamous cell carcinoma via regulation of the Wnt/beta-catenin and Hedgehog signaling pathways. Exp Cell Res 2017; 361: 170-177. https://doi. org/10.1016/j.yexcr.2017.10.016
[16] YANG K, DU J, SHI D, JI F, JI Y et al. Knockdown of MSI2 inhibits metastasis by interacting with caveolin-1 and inhibiting its ubiquitylation in human NF1-MPNST cells. Cell Death Dis 2020; 11: 489. https://doi.org/10.1038/s41419020-2703-x

[17] YANG C, ZHANG W, WANG L, KAZOBINKA G, HAN X et al. Musashi-2 promotes migration and invasion in bladder cancer via activation of the JAK2/STAT3 pathway. Lab Invest 2016; 96: 950-958. https://doi.org/10.1038/labinvest.2016.71

[18] MERCER TR, DINGER ME, MATTICK JS. Long non-coding RNAs: insights into functions. Nat Rev Genet 2009; 10: 155-159. https://doi.org/10.1038/nrg2521

[19] PONTING CP, OLIVER PL, REIK W. Evolution and functions of long noncoding RNAs. Cell 2009; 136: 629-641. https://doi.org/10.1016/j.cell.2009.02.006

[20] ZHANG EB, YIN DD, SUN M, KONG R, LIU XH et al. P53regulated long non-coding RNA TUG1 affects cell proliferation in human non-small cell lung cancer, partly through epigenetically regulating HOXB7 expression. Cell Death Dis 2014; 5: e1243. https://doi.org/10.1038/cddis.2014.201

[21] OZGUR E, MERT U, ISIN M, OKUTAN M, DALAY N et al. Differential expression of long non-coding RNAs during genotoxic stress-induced apoptosis in HeLa and MCF-7 cells. Clin Exp Med 2013; 13: 119-126. https://doi.org/10.1007/ s10238-012-0181-X

[22] HAN Y, LIU Y, GUI Y, CAI Z. Long intergenic non-coding RNA TUG1 is overexpressed in urothelial carcinoma of the bladder. J Surg Oncol 2013; 107: 555-559. https://doi. org/10.1002/jso.23264

[23] ZHOU H, SUN L, WAN F. Molecular mechanisms of TUG1 in the proliferation, apoptosis, migration and invasion of cancer cells. Oncol Lett 2019; 18: 4393-4402. https://doi. org/10.3892/ol.2019.10848

[24] MA L, SUN X, KUAI W, HU J, YUAN Y et al. LncRNA SOX2 overlapping transcript acts as a miRNA sponge to promote the proliferation and invasion of Ewing's sarcoma. Am J Transl Res 2019; 11: 3841-3849.

[25] BARTEL DP. MicroRNAs: genomics, biogenesis, mechanism, and function. Cell 2004; 116: 281-297. https://doi. org/10.1016/s0092-8674(04)00045-5

[26] FABRIS L, CEDER Y, CHINNAIYAN AM, JENSTER GW, SORENSEN KD et al. The Potential of MicroRNAs as Prostate Cancer Biomarkers. Eur Urol 2016; 70: 312-322. https:// doi.org/10.1016/j.eururo.2015.12.054

[27] FERRACIN M, VERONESE A, NEGRINI M. Micromarkers: miRNAs in cancer diagnosis and prognosis. Expert Rev Mol Diagn 2010; 10: 297-308. https://doi.org/10.1586/ erm.10.11

[28] YU SL, CHEN HY, YANG PC, CHEN JJ. Unique MicroRNA signature and clinical outcome of cancers. DNA Cell Biol 2007; 26: 283-292. https://doi.org/10.1089/dna.2006.0555

[29] DE FEO A, SCIANDRA M, FERRACIN M, FELICETTI F, ASTOLFI A et al. Exosomes from CD99-deprived Ewing sarcoma cells reverse tumor malignancy by inhibiting cell migration and promoting neural differentiation. Cell Death Dis 2019; 10: 471. https://doi.org/10.1038/s41419019-1675-1 
[30] JEMAL A, BRAY F, CENTER MM, FERLAY J, WARD E et al. Global cancer statistics. CA Cancer J Clin 2011; 61: 69-90. https://doi.org/10.3322/caac.20107

[31] BALAMUTH NJ, WOMER RB. Ewing's sarcoma. Lancet Oncol 2010; 11: 184-192. https://doi.org/10.1016/S14702045(09)70286-4

[32] MA M, PEI Y, WANG X, FENG J, ZHANG Y et al. LncRNA XIST mediates bovine mammary epithelial cell inflammatory response via NF-kappaB/NLRP3 inflammasome pathway. Cell Prolif 2019; 52: e12525. https://doi.org/10.1111/ cpr. 12525

[33] ZHANG Q, GENG PL, YIN P, WANG XL, JIA JP et al. Down-regulation of long non-coding RNA TUG1 inhibits osteosarcoma cell proliferation and promotes apoptosis. Asian Pac J Cancer Prev 2013; 14: 2311-2315. https://doi. org/10.7314/apjcp.2013.14.4.2311

[34] ZHOU Y, LU Y, LI R, YAN N, LI X et al. Prognostic role of long non-coding RNA TUG1 expression in various cancers: a meta-analysis. Oncotarget 2017; 8: 100499-100507. https:// doi.org/10.18632/oncotarget.20037

[35] LI N, SHI K, KANG X, LI W. Prognostic value of long noncoding RNA TUG1 in various tumors. Oncotarget 2017; 8: 65659-65667. https://doi.org/10.18632/oncotarget.20025

[36] HOU G, WANG Y, ZHANG M, HU Y, ZHAO Y et al. miR199a-3p suppresses progression of esophageal squamous cell carcinoma through inhibiting mTOR/p70S6K pathway. Anticancer Drugs 2021; 32: 157-167. https://doi.org/10.1097/ CAD.0000000000000983
[37] BAI J, JIAO WY. Down-Regulation of ZEB1 by miR-199a3p Overexpression Restrains Tumor Stem-Like Properties and Mitochondrial Function of Non-Small Cell Lung Cancer. Onco Targets Ther 2020; 13: 4607-4616. https://doi. org/10.2147/OTT.S244525

[38] DONG P, XIONG Y, HANLEY SJB, YUE J, WATARI H. Musashi-2, a novel oncoprotein promoting cervical cancer cell growth and invasion, is negatively regulated by $\mathrm{p} 53$ induced miR-143 and miR-107 activation. J Exp Clin Cancer Res 2017; 36: 150. https://doi.org/10.1186/s13046-0170617-y

[39] TAY Y, RINN J, PANDOLFI PP. The multilayered complexity of ceRNA crosstalk and competition. Nature 2014; 505 : 344-352. https://doi.org/10.1038/nature12986

[40] ZHAO W, JIANG X, YANG S. lncRNA TUG1 Promotes Cell Proliferation, Migration, and Invasion in Hepatocellular Carcinoma via Regulating miR-29c-3p/COL1A1 Axis. Cancer Manag Res 2020; 12: 6837-6847. https://doi.org/10.2147/ CMAR.S256624

[41] ZHANG K, ZHOU H, YAN B, CAO X. TUG1/miR-133b/ CXCR4 axis regulates cisplatin resistance in human tongue squamous cell carcinoma. Cancer Cell Int 2020; 20: 148. https://doi.org/10.1186/s12935-020-01224-9 